\title{
Novel Tool for the Study of Cholecystokinin-stimulated Pancreatic Enzyme Secretion
}

Herbert Y. Gaisano, Ulrich G. Klueppelberg, Delia I. Pinon, Michael A. Pfenning, ${ }^{\star}$ Stephen P. Powers, and Laurence J. Miller Gastroenterology Research Unit and the ${ }^{*}$ Department of Pharmacology, Mayo Clinic and Foundation, Rochester, Minnesota 55905

\begin{abstract}
The molecular events that mediate cholecystokinin (CCK)stimulated pancreatic secretion are not well defined because of the complex receptor-binding and concentration-response characteristics of this hormone. Functional models of receptor occupancy initiating the cascade leading to secretion have been complicated by the inhibition of secretion effected by supramaximal concentrations of CCK. Recent report of a CCK analogue that does not exhibit supramaximal inhibition led us to synthesize a similar analogue that could also be radiolabeled for studies of receptor binding and affinity labeling, and for studies of second messenger activity. This probe, D-Tyr-Gly[(Nle $\left.{ }^{28,31}\right)$ CCK-26-32]-phenethyl ester, was a fully efficacious secretagogue with no supramaximal inhibition, and, unlike native hormone, bound to a single class of sites present on both acini and membranes. Occupation of this site correlated well with stimulation of secretion. Evidence that this was indeed a CCK-binding site were the abilities of CCK and the antagonist L-364, 718 to inhibit binding of this analogue. Affinity labeling confirmed the identity of the site mediating secretory stimulation as a $M_{r}=85,000-95,000$ protein. Whereas the nonhydrolyzable guanosine triphosphate analogue, 5 '-guanylyl-imidodiphosphate, was a potent inhibitor of CCK binding, it had no effect on binding of this secretagogue, suggesting that a novel cascade not involving a guanine nucleotide-binding protein mediates CCK stimulation of pancreatic secretion.
\end{abstract}

\section{Introduction}

Cholecystokinin (CCK) is a gastrointestinal peptide hormone that is the predominant physiological stimulant of pancreatic enzyme secretion. However, our understanding of the molecular events mediating this process is limited because of the complex receptor-binding and concentration-response characteristics of this hormone and our inability to correlate the occupancy of a binding site with intracellular events leading to enzyme secretion.

\footnotetext{
Address reprint requests to Dr. Laurence J. Miller, Gastroenterology Research Unit, Mayo Clinic, Rochester, MN 55905.

Received for publication 24 August 1988 and in revised form 21 September 1988.
}

J. Clin. Invest.

(C) The American Society for Clinical Investigation, Inc. $0021-9738 / 89 / 01 / 0321 / 05 \quad \$ 2.00$

Volume 83, January 1989, 321-325
CCK has an unusual concentration-response relationship, in which increasing concentrations of hormone stimulate pancreatic secretion up to a maximum, whereas supramaximal concentrations inhibit secretion (1). To explain this, a model has been proposed in which two classes of hormone binding sites exist, a high-affinity site that stimulates secretion and a low-affinity site that inhibits secretion (1). Indeed, direct CCK radioligand binding to pancreatic acini have demonstrated two classes of binding sites (1); ligands selective for each class, however, have not been described. In addition, only a single class of CCK-binding sites has been observed on pancreatic membranes (2). Whereas this site has been biochemically characterized by affinity labeling (3-5), the relationship between this site and those present on acini is unclear, and it has been debated whether this protein represents the receptor that mediates CCK-stimulated enzyme secretion.

Several pancreatic intracellular events involving a guanine nucleotide-binding protein, phospholipase C, inositol lipids, intracellular calcium, and protein kinases have been described to occur in response to CCK (6). It is unclear which of these is related to occupancy of each binding site, and which truly mediate enzyme secretion. It is currently generally accepted that the proximal events in this cascade include association and activation of a guanine nucleotide-binding protein, activation of phospholipase $C$, and stimulation of inositol lipid metabolism (6).

In this work, we report a unique analogue of CCK that facilitates understanding of this process by binding to a single site, affinity labeling a single binding protein, and exhibiting simpler biological characteristics in which stimulation of amylase secretion occurs without the supramaximal inhibition of secretion typical of CCK. In addition, occupation of a binding site by this analogue can be closely correlated with its stimulation of secretion. Note that stimulation of pancreatic enzyme secretion by this CCK analogue occurs without the mediation of a guanine nucleotide-binding protein, suggesting that CCK stimulates pancreatic secretion via an additional, novel cascade.

\section{Methods}

Peptides. Synthetic native CCK-8 (CCK-26-33) was the kind gift of Dr. M. A. Ondetti, Squibb Institute for Medical Research (Princeton, NJ). We synthesized analogues of this peptide, D-Tyr-Gly-[(Nle $\left.{ }^{28,31}\right) \mathrm{CCK}-$ 26-33] and ${ }^{125} \mathrm{I}-\mathrm{D}-\mathrm{Tyr}-\mathrm{Gly}\left[\left(\mathrm{Nle}^{28,31}, \mathrm{pNO}_{2}-\mathrm{Phe}^{33}\right) \mathrm{CCK}-26-33\right]$, which we have previously validated as having activities similar to native hormone, but having advantages for binding and affinity labeling studies $(3,4,7)$. 
Design of the new CCK receptor probe for this work was based on a report of a related pancreatic secretagogue that was deficient in CCK's ability to inhibit secretion when present in supramaximal concentrations (8). That peptide had no site for radioiodination, and was not useful for studies of binding or affinity labeling. We therefore synthesized ${ }^{125} \mathrm{I}-\mathrm{D}-\mathrm{Tyr}-\mathrm{Gly}-\left[\left(\mathrm{Nle}^{28,31}\right) \mathrm{CCK}-26-32\right]-$ phenethyl ester (OPE analogue) ${ }^{1}$ by solid-phase and solution techniques similar to those we have reported for the above peptides (7). However, synthesis was initiated by coupling the aspartic acid-phenethyl ester $\beta$-carboxyl group to the resin using an acid-labile spacer.

Peptides were radioiodinated using $N$-chloro-benzenesulfonamide and purified by HPLC to $2,000 \mathrm{Ci} / \mathrm{mmol}$, using the method we have previously described (3).

Tissue preparations. Enriched pancreatic plasma membranes were prepared from 125-150-g male Sprague-Dawley rats (3). Dispersed pancreatic acini were prepared from similar animals by sequential enzymatic and mechanical dissociation (9). Incubations were performed in Krebs-Ringers-Hepes medium, containing $25 \mathrm{mM}$ Hepes, pH 7.4, $104 \mathrm{mM} \mathrm{NaCl}, 5 \mathrm{mM} \mathrm{KCl}, 1.2 \mathrm{mM} \mathrm{MgSO}_{4}, 2 \mathrm{mM} \mathrm{CaCl}_{2}, 1$ $\mathrm{mM} \mathrm{KH}_{2} \mathrm{PO}_{4}, 2.5 \mathrm{mM}$ D-glucose, $0.2 \% \mathrm{BSA}, 0.01 \%$ soybean trypsin inhibitor, $1 \mathrm{mM}$ 2-mercaptoethanol, and $1 \mathrm{mM}$ PMSF.

Biological activity and binding studies. For biological activity studies, pancreatic acini $\left(10^{6}\right.$ cells $)$ were incubated with agonists for $40 \mathrm{~min}$ at $37^{\circ} \mathrm{C}$, and amylase was assayed $(2,10)$. For binding assays, membranes $(15-20 \mu \mathrm{g})$ or acini $\left(10^{6}\right.$ cells) were incubated with $8-10 \mathrm{pM}$ radioligand for $60 \mathrm{~min}$, at $25^{\circ} \mathrm{C}$ for membranes and at $37^{\circ} \mathrm{C}$ for acini, conditions demonstrated to be at steady state (3). Bound was separated from free radioligand by filtration on a cell harvester (Skatron, Inc., Sterling, VA). Nonspecific binding, determined in the presence of 1 $\mu \mathrm{M}$ CCK-26-33 or OPE-analogue, was $<15 \%$ of total binding. The data reported represent specific binding.

Affinity labeling. For affinity labeling, binding to membranes (50 $\mu \mathrm{g})$ or acini $\left(20 \times 10^{6}\right.$ cells) was performed as above, using $200 \mathrm{pM}$ radioligand. In photoaffinity labeling using D-Tyr-Gly[( $\mathrm{Nle}^{28,31}, \mathrm{pNO}_{2}-$ $\mathrm{Phe}^{33}$ )CCK-26-33], binding was performed in the dark, and crosslinking was induced by photolysis using a $200-\mathrm{W}$ medium pressure lamp (Hanovia; Ace Glass Co., Vineland, NJ) with a Pyrex filter for 30 minutes at $4^{\circ} \mathrm{C}(4)$. The other ligands required cross-linking with 50 $\mu \mathrm{M}$ disuccinimidyl suberate or $5 \mu \mathrm{M} m$-maleimidobenzoyl $N$-hydroxysuccinimide ester for $5 \mathrm{~min}$ at $4^{\circ} \mathrm{C}$. Labeled membranes were pelleted by centrifugation, solubilized and separated by SDS-PAGE, and visualized by autoradiography ( 3 ).

In some experiments, the affinity-labeled band was electroeluted and further characterized by deglycosylation with $3 \mathrm{U}$ endo- $\beta-N$-glucosaminidase $F$ (endo $F$ ) in $0.1 \mathrm{M}$ sodium phosphate, $\mathrm{pH} 6.2,50 \mathrm{mM}$ EDTA, $0.1 \% \mathrm{NP}-40,0.1 \% \mathrm{SDS}$, and $1 \mathrm{mM}$ 2-mercaptoethanol at $37^{\circ} \mathrm{C}$ for $12 \mathrm{~h}(11)$.

\section{Results}

Indeed, D-Tyr-Gly-[(Nle $\left.{ }^{28,31}\right)$ CCK-26-32]-phenethyl ester stimulated amylase secretion with equal efficacy but lower potency $\left(\mathrm{ED}_{50}=2.5 \mathrm{nM}\right)$ than native $\mathrm{CCK}-8\left(\mathrm{ED}_{50}=0.014 \mathrm{nM}\right)$; and, unlike CCK-8, this analogue did not exhibit supramaximal inhibition of secretion (Fig. 1). Mixing experiments demonstrated that the OPE-analogue affected both components of the CCK-8 concentration-response curve. Added to submaximal concentrations of CCK-8, it increased secretion in a concentration-dependent manner to the same maximum as stimulated by CCK-8 alone. Added to supramaximal concentra-

1. Abbreviations used in this paper: Endo $\mathrm{F}$, endo- $\beta-N$-glucosaminidase F; Gpp(NH)p, 5'-guanylyl-imidodiphosphate; OPE-analogue, D-Tyr-Gly-[(Nle $\left.{ }^{28,31}\right)$ CCK-26-32]-phenethyl ester.

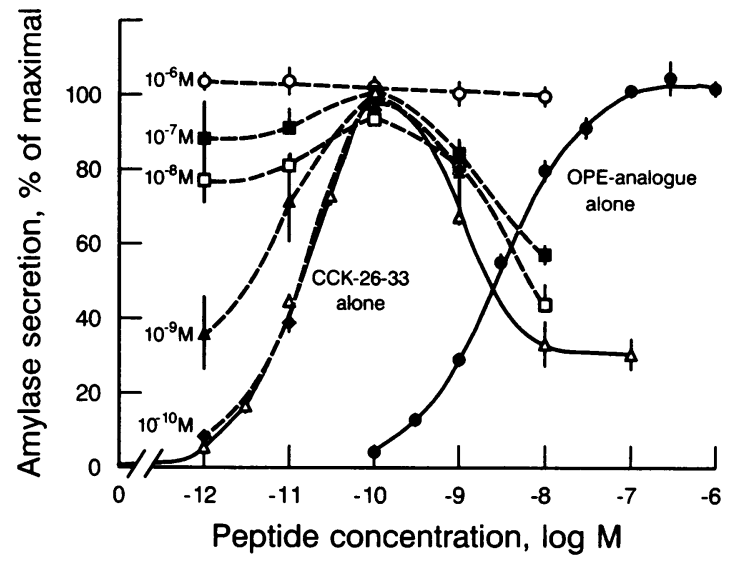

Figure 1. Stimulation of pancreatic amylase secretion by various concentrations of native $\mathrm{CCK}$ and the OPE-analogue alone or in combination. Dashed lines represent the addition of given concentration of OPE-analogue to CCK-26-33. Each point represents the mean \pm SEM of a minimum of four experiments performed in duplicate.

tions of CCK-8, it resulted in a concentration-dependent reversal of the CCK-8-induced inhibition of secretion.

The radioiodinated OPE-analogue bound in a saturable manner to both pancreatic acini and membranes, with characteristics similar in both $\left(K_{d}=16 \mathrm{nM}\right)$ (Fig. $\left.2 A\right)$. Computer analysis of these data using the LIGAND program (12) best fit a single class of binding sites, and the Hill coefficient was 1.1. These data are also consistent with the OPE-analogue binding to two classes of sites with equal affinities. In contrast, ${ }^{125} \mathrm{I}-\mathrm{D}$ Tyr-Gly-[(Nle $\left.\left.{ }^{28,31}\right)-\mathrm{CCK}-26-33\right]$ (previously shown to possess binding and biological activity identical to native CCK-8 [13]) had different binding characteristics to pancreatic acini and membranes, with analysis suggesting the presence of two sites with differing affinities on acini $\left(K_{\mathrm{d}_{1}}=0.144 \mathrm{nM}, K_{\mathrm{d}_{2}}=17 \mathrm{nM}\right.$; Hill coefficient $=0.3$ ) and only one site on plasma membranes $\left(K_{\mathrm{d}}=0.8 \mathrm{nM}\right)$ (Fig. $2 \mathrm{~B}$ ).

Evidence that the site that binds the OPE-analogue was indeed a CCK-binding site included the abilities of both this analogue and CCK to compete for binding of each other (Fig. 2, $C$ and $D$ ), and the ability of the specific CCK receptor antagonist L-364,718 (Merck, Sharp and Dohme, West Point, PA) to compete for binding of both (Fig. $2 E$ ). Interestingly, L-364,718 competition for binding of ${ }^{125} \mathrm{I}$-OPE-analogue to acini demonstrated a single binding site, whereas its competition for binding of the native CCK-radioligand demonstrated two classes of binding sites (Fig. $2 E$ ). Furthermore, close correlation existed between occupancy of the OPE-analoguebinding site and stimulation of pancreatic secretion, with $95 \%$ of sites occupied at maximal secretion (Figs. 1 and $2 A$ ), suggesting that this probe interacts with the receptor relevant in CCK-stimulated secretion in a manner that obeys the mass action law.

When acini were incubated with native CCK, a second class of binding sites was evident (Fig. 2, $B, C$, and $D$ ), and the OPE-analogue was also able to bind to that site (Fig. 2, $C$ and $D$ ). However, this analogue acted as a competitive antagonist at that site, reversing the supramaximal inhibition of enzyme secretion that has been correlated with its occupancy by CCK (1) (Fig. 1). 

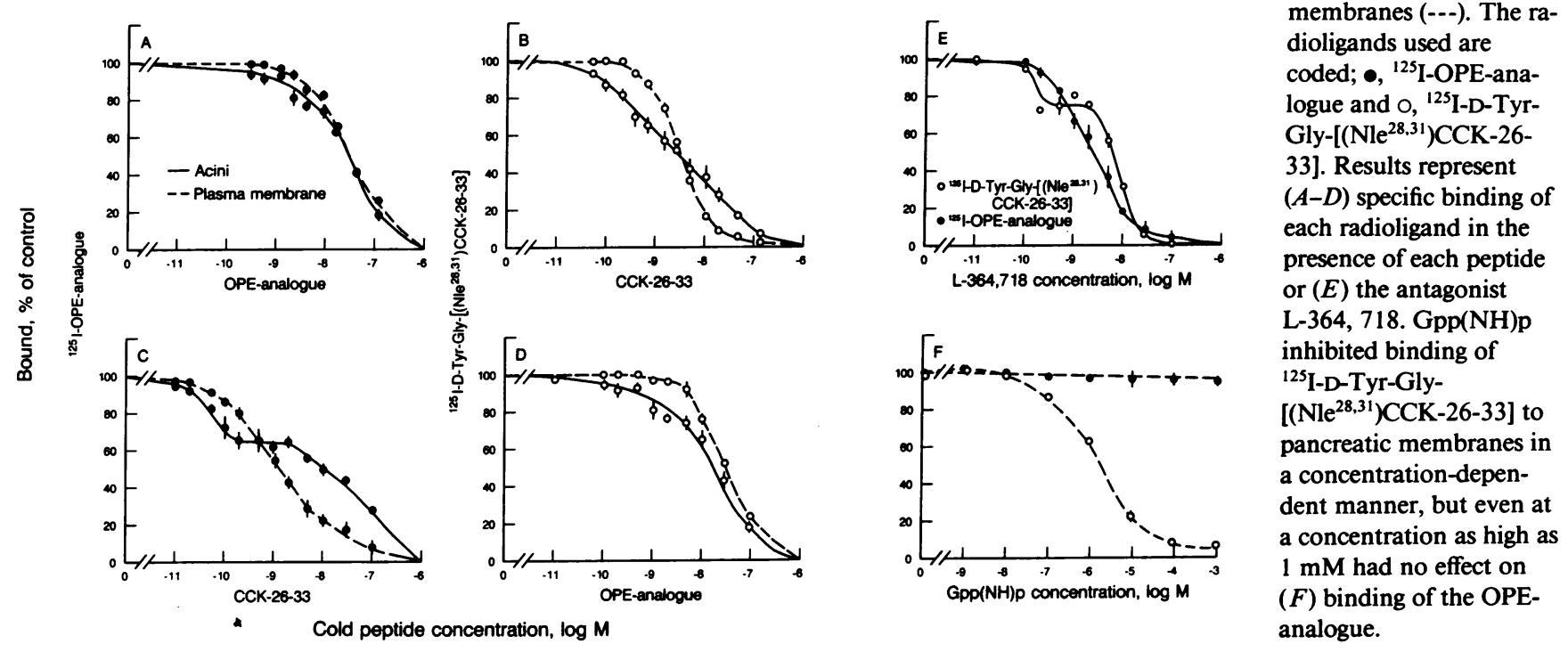

Figure 2. Competition binding studies with pancreatic acini (-) and membranes (---). The radioligands used are coded; •, ${ }^{125}$ I-OPE-analogue and $0,{ }^{125} \mathrm{I}-\mathrm{D}-\mathrm{Tyr}$ Gly-[(Nle $\left.{ }^{28,31}\right)$ CCK-26

33]. Results represent $(A-D)$ specific binding of each radioligand in the resence of each peptide inhibited binding of ${ }^{125}$ I-D-Tyr-Gly[(Nle $\left.\left.{ }^{28,31}\right) \mathrm{CCK}-26-33\right]$ to pancreatic membranes in a concentration-dependent manner, but even at a concentration as high as $(F)$ binding of the OPEanalogue.

The glycoprotein that bound the OPE-analogue on both acini and membranes was biochemically characterized by affinity labeling to be the same molecule labeled by a series of CCK receptor probes with sites of covalent attachment that span the receptor-binding domain $(3,4)$ (Fig. 3). ${ }^{125} \mathrm{I}-\mathrm{D}-\mathrm{Tyr}-$ Gly[(Nle $\left.\left.{ }^{28,31},-\mathrm{pNO}_{2}-\mathrm{Phe}^{33}\right) \mathrm{CCK}-26-33\right]$, a CCK receptor probe possessing a photolabile nitro-phenylalanyl moiety within the theoretical receptor-binding domain (4), labeled a $M_{\mathrm{r}}$ $=85,000-95,000$ protein on pancreatic membranes that was inhibited in a concentration-dependent manner by the OPEanalogue. Radiolabeled OPE-analogue, when used with disuccinimimidyl suberate (Fig. 3) or $m$-maleimidobenzoyl $N$-hydroxysuccinimide ester (data not shown), labeled the same $M_{\mathrm{r}}$ $=85,000-95,000$ protein on pancreatic membranes and acini as that labeled by the CCK receptor probe, and this was also inhibited in a concentration-dependent manner by CCK-8 or by the unlabeled OPE-analogue. Using disuccinimidyl suberate, an additional band of $M_{\mathrm{r}}=52,000$ was saturably labeled; however, this was not consistently seen and was much less prominent when using $m$-maleimidobenzoyl $N$-hydroxysuccinimide ester. The major $M_{\mathrm{r}}=85,000-95,000$ protein labeled by ${ }^{125} \mathrm{I}$-OPE-analogue was deglycosylated with endo $\mathrm{F}$ to yield the same $M_{\mathrm{r}}=42,000$ core protein as that labeled by a series of CCK-receptor probes (3) (Fig. 3). Staphylococcus aureus V8 protease peptide mapping of the labeled protein also confirmed this identity (data not shown).

The postulated proximal event in CCK-induced secretion is the association and activation of a guanine nucleotide-binding protein (14). The role of this protein can be demonstrated using the nonhydrolyzable guanosine triphosphate analogue, 5'-guanylyl-imidodiphosphate ( $\mathrm{Gpp}(\mathrm{NH}) \mathrm{p})$, to inhibit CCK binding and to increase dissociation of CCK from its receptor

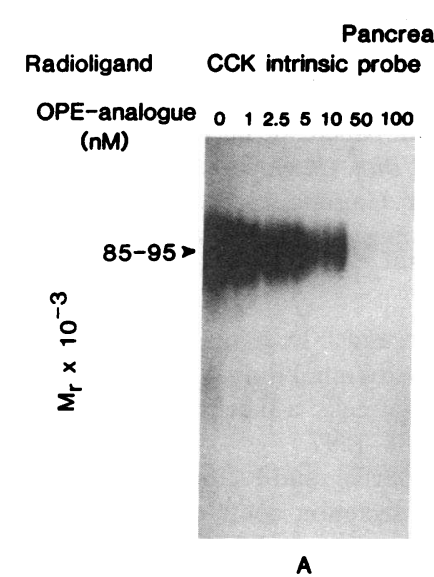

Pancreatic
probe

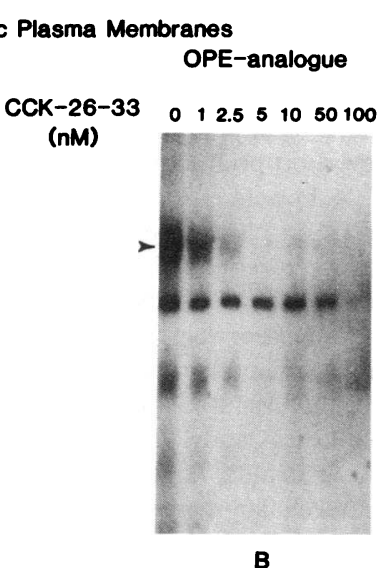

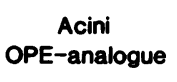

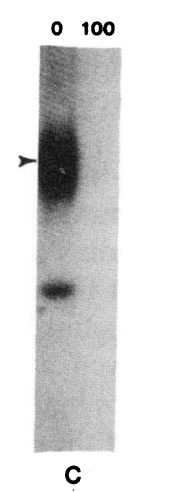

Figure 3. Affinity labeling of pancreatic plasma membranes and acini by the photolabile CCK receptor intrinsic probe, $(A)^{125} \mathrm{I}-\mathrm{D}-\mathrm{Tyr}-$ Gly[( $\left.\mathrm{Nle}^{28,31}, \mathrm{pNO}_{2}-\mathrm{Phe}^{33}\right)$ CCK-26-33], and $(B-D)^{125} \mathrm{I}-$ OPE-analogue (with disuccinimidyl suberate). Labeled membranes were solubilized and separated by SDS-PAGE $(10 \%$ gels for $A-C, 12.5 \%$ gel for $D$ ), and visualized by autoradiography. The same $M_{\mathrm{r}}=85,000$ 95,000 protein was specifically labeled by both radioligands on both membranes

and acini. It was inhibited by both peptides in a concentration-dependent manner. The ${ }^{125} \mathrm{I}$-OPE-analogue also labeled a $M_{\mathrm{r}}=67,000$ protein, presumably BSA, in a nonsaturable manner, and a minor $M_{\mathrm{r}}=52,000$ protein in a saturable manner. 
(15). Whereas native CCK clearly demonstrates this activity, no such event occurred under conditions in which the OPEanalogue stimulated enzyme secretion (Fig. $2 F$ ).

\section{Discussion}

We have designed and synthesized a radioiodinated analogue of CCK that is a fully efficacious stimulant of pancreatic secretion, but which, unlike native hormone, does not exhibit supramaximal inhibition of secretion. The OPE-analogue has allowed us to observe directly the functional characteristics of the binding site that mediates CCK-stimulated pancreatic secretion, and to biochemically characterize that site. The differences in binding and second messenger activity between this analogue and native CCK provide important insights into the mechanism of action of this hormone.

Unlike CCK, which binds to two classes of sites on acini, this analogue appears to bind to only one class of sites in the absence of CCK. Evidence that this site is indeed a CCK-binding site includes the abilities of both this analogue and CCK to compete for binding of each other, and the ability of the CCK receptor antagonist L364,718 to compete for binding of both. Of particular importance is the close correlation between occupancy of this site by the OPE-analogue and stimulation of enzyme secretion. This suggests that this probe interacts with the relevant receptor in CCK-stimulated secretion.

Biochemical characterization of the OPE-analogue-binding site using affinity-labeling techniques has identified a $M_{\mathrm{r}}$ $=85,000-95,000$ glycoprotein on both pancreatic acini and membranes. This is the same size as the protein that has been labeled by a series of CCK receptor probes with sites of covalent attachment that span the receptor-binding domain $(3,4)$. Further confirmation that the OPE-analogue-binding site is the same as that labeled by the other probes was its deglycosylation to the same-size core protein of $M_{\mathrm{r}}=42,000$, and its similar protease peptide mapping pattern.

These experiments also provide interesting insight into the second class of CCK-binding sites (low affinity), previously proposed as mediating inhibition of enzyme secretion (1). Although the OPE-analogue does not express supramaximal inhibition of secretion, it is able to bind to this site, where it behaves as a competitive antagonist, reversing CCK-induced supramaximal inhibition of enzyme secretion. The different activities of CCK and the OPE-analogue provide an opportunity to sort out the intracellular events associated with activation of the high-affinity CCK-binding site which lead to stimulation of secretion, from those associated with activation of the low-affinity CCK-binding site that lead to inhibition of secretion.

Note that the second class of CCK-binding sites is only observed on intact cells and not on plasmalemmal membranes from those cells. Also, intact cells express only a single binding site when incubated at $4^{\circ} \mathrm{C}(16)$. This may suggest that the expression of the second class of CCK-binding sites may actually depend on a temperature-dependent intracellular event. Perhaps those events that are stimulated by CCK, but not by the OPE-analogue, may be responsible for expression of the low-affinity site on the cell surface, or even for induction of a change in the affinity of the high-affinity binding site. The latter possibility may explain why only a single molecule has been affinity labeled by the most ideal CCK-based receptor probes, which are covalently attached via functional groups near or within the receptor-binding domain $(3,4)$.

The postulated proximal event in CCK-induced pancreatic activity is the association and activation of a guanine nucleotide-binding protein (14). The role of this protein can be demonstrated experimentally by incubation with the nonhydrolyzable guanosine triphosphate analogue, 5'-guanylyl-imidodiphosphate, which inhibits CCK binding and increases dissociation of CCK from its receptor (15). Whereas native CCK clearly induces guanine nucleotide-binding protein activity, no such event seems to occur under conditions that the OPE-analogue stimulates maximal enzyme secretion. This suggests the presence of a novel intracellular pathway not previously recognized to mediate CCK-stimulated pancreatic enzyme secretion. The recent observation that another related OPE-analogue of CCK does not induce phosphatidyl inositol hydrolysis, a more distal event in this postulated cascade, is consistent with this (8).

Thus, this unique CCK analogue provides evidence that the $M_{\mathrm{r}}=85,000-95,000$ pancreatic plasma membrane protein represents the proximal step in stimulus-secretion coupling. The previously proposed pathway of guanine nucleotide-binding protein association and activation leading to activation of phospholipase $\mathrm{C}$ and inositol lipid metabolism is not activated by this secretagogue, and may mediate CCK-induced supramaximal inhibition of secretion rather than CCK-stimulated pancreatic secretion. The mechanism of this stimulation is yet to be determined.

\section{Acknowledgments}

The authors thank Dr. Elliott Richelson for helpful discussions, Elizabeth Hadac for excellent technical assistance, and Marilyn LeQve for secretarial help.

This work was supported by National Institutes of Health grants, DK-32878 and DK-34988.

\section{References}

1. Jensen, R. T., G. F. Lemp, and J. D. Gardner. 1982. Interactions of $\mathrm{COOH}$-terminal fragments of cholecystokinin with receptors on dispersed acini from guinea pig pancreas. J. Biol. Chem. 257:55545559.

2. Miller, L. J., S. A. Rosenzweig, and J. D. Jamieson. 1981. Preparation and characterization of a probe for the cholecystokinin octapeptide receptor, $\mathrm{N} \alpha\left({ }^{125} \mathrm{I}\right.$-desaminotyrosyl)CCK-8, and its interactions with pancreatic acini. J. Biol. Chem. 256:12417-12423.

3. Pearson, R. K., and L. J. Miller. 1987. Affinity labeling of a novel cholecystokinin binding protein in rat pancreatic plasmalemma using new short probes for the receptor. J. Biol. Chem. 262:869-876.

4. Powers, S. P., D. Fourmy, H. Y. Gaisano, and L. J. Miller. 1988. Intrinsic photoaffinity-labeling probes for cholecystokinin-gastrin family receptors. D-Tyr-Gly[(Nle $\left.\left.{ }^{28,31}, \mathrm{pNO}_{2}-\mathrm{Phe}^{33}\right) \mathrm{CCK}-26-33\right]$. $J$. Biol. Chem. 263:5295-5300.

5. Rosenzweig, S. A., L. J. Miller, and J. D. Jamieson. 1983. Identification and localization of cholecystokinin binding sites on rat pancreatic plasma membranes and acinar cells: a biochemical and morphological study. J. Cell Biol. 96:1288-1297.

6. Schultz, I., H. Streb, E. Bayerdorffer, and K. Imamura, J. 1986. Intracellular messengers in stimuli-secretion coupling of pancreatic acinar cells. Cardiovasc. Pharmacol. 8 (Suppl. 8):S91-S96.

7. Powers, S. P., D. I. Pinon, and L. J. Miller. 1988. Use of $N$, $O$-bis-Fmoc-D-Tyr-ONSu for introduction of an oxidative iodination site into cholecystokinin family peptides. Int. J. Pept. Protein Res. 31:429-434. 
8. Lignon, M., M. Galas, M. Rodriquez, G. Gaillon, and J. Martinez. 1987. A CCK-analogue that exhibits part of the CCK response on rat pancreatic acini: relationships among amylase release, receptor affinity and phosphoinositide breakdown. In Gastrin and Cholecystokinin. Chemistry, Physiology and Pharmacology. J.-P. Bali, and J. Martinez, editors. Elsevier Science Publishers, New York. 57-60.

9. Schultz, G. S., M. P. Sarras, G. R. Gunther, B. E. Hull, H. A. Alicea, F. S. Gorelick, and J. D. Jamieson. 1980. Guinea pig pancreatic acini prepared with purified collagenase. Exp. Cell Res. 130:49-62.

10. Bernfeld, P. 1951. Amylases, $\alpha$ and $\beta$. Adv. Enzymol. 12:379428.

11. Pearson, R. K., L. J. Miller, E. M. Hadac, and S. P. Powers. 1987. Analysis of the carbohydrate composition of the pancreatic plasmalemmal glycoprotein affinity labeled by short probes for the cholecystokinin receptor. J. Biol. Chem. 262:13850-13856.

12. Munson, P. J., and D. Rodbard. 1980. LIGAND: a versatile computerized approach for characterization of ligand-binding systems. Anal. Biochem. 107:220-239.

13. Pearson, R. K., S. P. Powers, E. M. Hadac, H. Y. Gaisano, and L. J. Miller, 1987. Establishment of a new short, protease-resistant, affinity labeling reagent for the cholecystokinin receptor. Biochem. Biophys. Res. Commun. 147:346-353.

14. Merritt, J. E., C. W. Taylor, R. P. Rubin, and V. W. Putney, Jr. 1986. Evidence suggesting that a novel guanine nucleotide regulatory protein couples receptors to phospholipase $\mathrm{C}$ in exocrine pancreas. Biochem. J. 236:337-343.

15. Williams, J. A., and D. J. McChesney. 1987. Cholecystokinin induces the interaction of its receptor with a guanine nucleotide binding protein. Regul. Pept. 18:109-117.

16. Wank, S. A., R. T. Jensen, and J. D. Gardner. 1988. Kinetics of binding of cholecystokinin to pancreatic acini. Am. J. Physiol. 255:G106-G112. 